\section{Self-Rated Oral Health and Dental Service Use of Older Korean Americans with Diabetes}

\author{
Yuri Jang ${ }^{1,2 \star}$, Min-Kyoung Rhee ${ }^{3}$, Chung Hyeon Jeong ${ }^{2}$, Roseann \\ Mulligan $^{4}$ and Miyong T Kim ${ }^{5}$
}

${ }^{1}$ Edward R Roybal Institute on Aging, University of Southern California, Los Angeles, USA

${ }^{2}$ Suzanne Dworak-Peck School of Social Work, University of Southern California, Los Angeles, USA

${ }^{3}$ Leonard Davis School of Gerontology, University of Southern California, Los Angeles, USA

${ }^{4}$ Herman Ostrow School of Dentistry, University of Southern California, Los Angeles, USA

${ }^{5}$ School of Nursing, The University of Texas at Austin, Texas, USA

\begin{abstract}
The study examined the effect of diabetes on self-rated oral health and dental service use in older Korean Americans. Surveys were administered to Korean Americans aged 60 and older $(N=2,138)$ in five cities. Logistic regression models were used to assess the effect of the presence of diabetes on self-rated oral health and dental service use, after controlling for demographic, immigration-related and health and access variables. More than $61 \%$ of those affected by diabetes rated their oral health as either fair or poor; however, almost $40 \%$ had never visited a dental clinic in the past year. The presence of diabetes significantly increased the odds of reporting fair or poor oral health but had no significant effect on dental service use. The findings underscore unmet dental care needs among older adults with diabetes and call for the need of integrated care for diabetes and oral health.
\end{abstract}

Keywords: Dental service use; Diabetes; Older koreanamericans; Oral health

*Corresponding author: Yuri Jang, Edward R Roybal Institute on Aging, Suzanne Dworak-Peck School of Social Work, University of Southern California 1150 S. Olive Street, Suite 1400, Los Angeles, USA, Tel: +1 2138216441; E-mail: yurij@usc.edu

Citation: Jang Y, Rhee MK, Jeong CH, Mulligan R, Kim (2019) Self-Rated Oral Health and Dental Service Use of Older Korean Americans with Diabetes. J Gerontol Geriatr Med 5: 034.

Received: July 16, 2019; Accepted: July 23, 2019; Published: July 30, 2019

Copyright: $\odot 2019$ Jang $Y$, et al. This is an open-access article distributed under the terms of the Creative Commons Attribution License, which permits unrestricted use, distribution, and reproduction in any medium, provided the original author and source are credited.

\section{Introduction}

Diabetes poses a critical risk to oral health as it can cause dry mouth (salivary hypofunction) that often precedes caries of the teeth and problems with gingival tissue (gums) [1-5]. Particularly the bidirectional relationship between glycemic levels and periodontal disease has been widely known [6-8]. Nevertheless, dental care has not been sufficiently addressed in diabetes care regimens $[9,10]$ and many individuals with diabetes and their caregivers are unaware of diabetes-related oral manifestations and complications [10-12]. Furthermore, dental health professionals are generally absent from diabetes management teams, often leaving patients' oral health needs unattended $[9,10]$.There is a long-standing concern that the medical-dental divide in the USA healthcare system exacerbates the lack of coordination between oral health and diabetes care [13-15].

Given the compelling need to build a foundation for integrating diabetes care and oral health, the present study examined how the presence of diabetes affect self-rated oral health and dental service use in older Korean Americans. This population is appropriate to target for several reasons: (a) they are one of the rapidly expanding immigrant groups and they are the fifth largest Asian American subgroup $[16,17]$; (b) they manifest marked disparities in health and healthcare[18]; and (c) their prevalence of diabetes and unmet needs for dental care are high [19-21]. Despite their low rate of traditional risk factors for diabetes such as obesity, Koreans have a high prevalence of diabetes. Although the precise reasons for this are not clear, a genetic predisposition toward high central adiposity and dysfunction of $\beta$-cells in the pancreas have been discussed as potential contributing factors [22]. A sizable body of literature has examined physical and mental health implications of diabetes in older Korean Americans, $[20,21]$ but attention has rarely been paid to oral health arena.

In the present study, two oral health-related variables are of interest: self-rated oral health and dental service use. Given its high correlations with the results of clinical oral examination (e.g., the numbers of decayed, missing and filled teeth), self-rated oral health is considered an efficient indicator of oral health needs [23]. Despite the preventive benefits of a regular dental visit, however, a substantial proportion of the USA population faces barriers to receiving dental care [24]. This limited access to dental care is of particular concern for individuals with heightened oral health risk, such as patients with diabetes. Because unattended oral health problems lead to complications with diabetes and soaring healthcare expenditures $[9,25]$ efforts to promote the dental care of individuals with diabetes are critical.

The present study was undertaken to facilitate those efforts by exploring the status of oral health and dental service use of older Korean Americans with diabetes and examining how the presence of diabetes may affect their self-perceptions of oral health and use of dental services. We hypothesized that diabetes would have a negative impact on self-ratings of oral health but that it would not necessarily predict the use of dental care services. We conducted this assessment of oral health needs and service use with consideration of demographic variables, immigration-related variables and health and access variables. 


\section{Methods}

\section{Participants}

Data were drawn from the Study of Older Korean Americans (SOKA), a survey of Korean immigrants aged 60 and older conducted in five cities: Los Angeles, New York City, Austin, Honolulu and Tampa. Community-based samples were recruited by a team of investigators who shared the target population's language and culture. At each site, the project began by compiling a database listing Korean-oriented resources, services and amenities; this databasenot only facilitated the research team's efforts for community engagement but also guided the selection of potential survey locations.In the development and use of these ethnic resource databases, community advisors' input was actively solicited. The surveys took place at multiple locations and events (e.g., churches, temples, grocery stores, small group meetings and cultural events) from April 2017 to February 2018. The survey instrument consisted of a standardized questionnaire in Korean. Developed through a back-translation and reconciliation method, the survey was designed to be self-administered, but trained interviewers were available for anyone who needed assistance.It took respondents about 40 minutes to complete the 12-page questionnaire, and they were each paid USA $\$ 20$ for participation. The project was approved by a university Institutional Review Board. After removal of those with more than $10 \%$ missing responses on the entire questionnaire $(\mathrm{n}=26)$ and no response to the question on diabetes $(\mathrm{n}=$ 12 ), the final sample consisted of 2,138 participants.

\section{Measures}

\section{Self-rated oral health}

Participants were asked how they would rate their overall oral health condition on a 5-point scale. Responses were then dichotomized into excellent/verygood/good (0) and fair/poor (1).

\section{Dental service use}

Participants were also asked how many times they had visited a dentist in the past 12 months. Responses were recoded into a binary variable $(0=$ no use, $1=$ use $)$.

\section{Diabetes}

The presence of diabetes was assessed by asking participants whether their medical doctor had informed them that they had diabetes.Responses were coded as yes (1) or no (0). The type of diabetes (Type 1 or 2) was not assessed.

\section{Demographic variables}

Backgroundinformation included age (in years), gender $(0=$ male, $1=$ female $)$, marital status $(0=$ married, $1=$ not married $)$ and education $(0=\leq$ high school graduation, $1=>$ high school graduation $)$.

\section{Immigration-related variables}

Participants were asked how long they had lived in the USA with responses coded in years. Acculturation was assessed with a 12-item acculturation inventory [26] that included questions on language use, media consumption, food consumption, social relations, sense of belonging and familiarity with culture. The total score could range from 0 to 36 , with higher scores indicating a greater level of acculturation to mainstream American culture. Validation of the instrument has been conducted in previous studies with older Korean Americans [26]. Internal consistency of the scale in the present sample was high $(\alpha=$ $0.91)$.

\section{Health and access variables}

For medical comorbidity, the presence of medical conditions other than diabetes was used. The list included 9 chronic diseases and conditions common in older populations: hypertension, heart disease, stroke, cancer, arthritis, hepatitis, kidney problem, asthma and chronic obstructive pulmonary disease. Each condition was coded using a yes/no format, with the total count used for analysis. As an access variable, dental insurance was coded as not insured (0) or insured (1).

\section{Analytical strategy}

Descriptive characteristics of the overall sample $(\mathrm{N}=2,138)$ were reviewed. Using $t$ or chi-square tests, we compared groups with $(\mathrm{n}=$ $588)$ and without $(\mathrm{n}=1,550)$ diabetes. Separate logistic regression models were performed to assess self-rated oral health and dental service use. For each outcome, an unadjusted model only with diabetes was tested, followed by an adjusted model with a control for demographic (age, gender, marital status and education), immigration-related (length of stay in the USA and acculturation), and health and access (medical comorbidity and dental insurance) variables. All analyses were performed using IBM SPSS Statistics 25 [27].

\section{Results}

\section{Sample characteristics and comparisons between individ- uals with and without diabetes}

Descriptive characteristics of the overall sample are summarized in Table 1. More than a quarter of the sample (27.5\%) had diabetes. The mean age of the overall sample was 73.4 years $(\mathrm{SD}=7.97)$, with a range from 60 to 100 .About $67 \%$ were women, more than $39 \%$ were not married and $40 \%$ had received more than a high school education. The number of years of residence in the USA averaged 31.5 (SD = 12.1 ; range $=0.17$ to 80 ); the mean score for acculturation was 12.2 $(\mathrm{SD}=7.08$; range $=0$ to 35$)$.

Table 1 also presents comparisons between those with $(\mathrm{n}=588)$ and without $(\mathrm{n}=1,550)$ diabetes. The group with diabetes was more likely to be older, unmarried and less educated. Although those with diabetes had stayed longer in the USA, their level of acculturation was lower than that of their non-diabetic counterparts. The group with diabetes also showed a higher number of comorbid conditions. At $61.3 \%$, the rate of reporting fair/poor oral health was substantially higher in the group with diabetes. Ranging from $61.1 \%$ to $62.5 \%$, the rate of dental service use was comparable between the groups. It is alarming that almost $40 \%$ of the individuals affected by diabetes had never visited a dental clinic in the past year.

\section{The effect of diabetes on self-rated oral health and dental service use}

Table 2 summarizes the logistic regression models of self-rated oral health and dental service use. In the unadjusted model, the presence of diabetes increased the odds of reporting fair/poor oral health by 1.74 times. The odds remained significant in the adjusted model. All covariates except age were significant. 
Citation: Jang Y, Rhee MK, Jeong CH, Mulligan R, Kim (2019) Self-Rated Oral Health and Dental Service Use of Older Korean Americans with Diabetes. J Gerontol Geriatr Med 5: 034.

\begin{tabular}{|l|c|c|c|}
\hline & Overall sample & Individuals without diabetes $(\boldsymbol{n}=\mathbf{1 , 5 5 0})$ & Individuals with diabetes $(\boldsymbol{n}=\mathbf{5 8 8})$ \\
\hline Diabetes & $27.5 \%$ & - & - \\
\hline Age (years, M \pm SD) & $73.4 \pm 7.97$ & $72.8 \pm 8.02$ & $74.9 \pm 7.61$ \\
\hline Gender (female) & $66.7 \%$ & $66.9 \%$ & $66.3 \%$ \\
\hline Marital status (not married) & $39.4 \%$ & $37.6 \%$ & $44.0 \%$ \\
\hline Education (>high school graduation) & $40.0 \%$ & $41.8 \%$ & $(0.06)$ \\
\hline Length of stay in the U.S. (years, M \pm SD) & $31.5 \pm 12.1$ & $31.1 \pm 12.2$ & $(7.09 * *)$ \\
\hline Acculturation (M \pm SD) & $12.2 \pm 7.08$ & $12.6 \pm 7.11$ & $(7.77 * *)$ \\
\hline Medical comorbidity (M \pm SD) & $1.29 \pm 1.23$ & $1.12 \pm 1.13$ & $2.35 *$ \\
\hline Dental insurance & $29.2 \%$ & $28.7 \%$ & $11.1 \pm 6.86$ \\
\hline Self-rated oral health (fair/poor) & $52.5 \%$ & $49.2 \%$ & $1.74 \pm 1.38$ \\
\hline Dental service use & $62.1 \%$ & $62.5 \%$ & $29.4 \%$ \\
\hline
\end{tabular}

Table 1: Sample descriptions and comparisons between individuals with and without diabetes.

$* \mathrm{p}<0.05 . * * \mathrm{p}<0.01 . * * * \mathrm{p}<0.001$

\begin{tabular}{|l|c|c|c|}
\hline & \multicolumn{2}{|c|}{ Odds Ratio (95\% Confidence Interval) } \\
\hline & \multicolumn{2}{|c|}{ Self-rated Oral Health (Fair/poor) } & \multicolumn{2}{c|}{ Dental Service Use } \\
\hline Diabetes & $1.74^{* * *}(1.43,2.14)$ & $1.41^{* *}(1.13,1.76)$ & $0.95(0.77,1.16)$ \\
\hline Age & $1.01(0.99,1.02)$ & $0.98(0.97,1.00)$ \\
\hline Female & $0.58^{* * *}(0.47, .74)$ & $1.30^{*}(1.05,1.62)$ \\
\hline Not married & $1.45^{* *}(1.16,1.79)$ & $0.76^{*}(0.62,0.94)$ \\
\hline$>$ High school graduation & $0.55^{* * *}(0.44,0.68)$ & $1.49^{* * *}(1.20,1.85)$ \\
\hline Length of stay in the U.S. & & $0.98^{*}(0.97,0.99)$ & $1.02^{* * *}(1.01,1.03)$ \\
\hline Acculturation & $0.93^{* * *}(0.91,0.95)$ & $1.00(0.98,1.02)$ \\
\hline Medical comorbidity & $1.27^{* *}(1.15,1.38)$ & $1.01(0.92,1.09)$ \\
\hline Dental insurance & $0.75^{*}(0.61,0.93)$ & \\
\hline
\end{tabular}

Table 2: Regression models of self-rated oral health and dental service use.

${ }^{*} \mathrm{p}<0.05 .{ }^{* *} \mathrm{p}<0.01 . * * * \mathrm{p}<0.001$

The higher odds of reporting fair/poor oral health were associated with male gender, unmarried status, lower education, fewer years of USA residence, lower levels of acculturation, higher numbers of comorbid conditions and lack of dental insurance.

With regard to dental service use, the presence of diabetes showed no significant effect in both the unadjusted and adjusted models. The higher odds of using dental services were associated with female gender, married status, higher education, longer years of USA residence and dental insurance. It is particularly notable that the odds of using dental service were 2.34 times higher among those who were covered by dental insurance than among those who were not.

\section{Discussion}

Responding to the close link between diabetes and oral health problems,[1-3,5] the present study explored how self-rated oral health and dental service use of older Korean Americans might be influenced by the presence of diabetes. The findings of our analyses provide support to the proposed hypotheses that the presence of diabetes would be associated with heightened oral health needs (fair/poor ratings of oral health) but would not lead to increased use of dental services.

The prevalence rate of diabetes in the present sample was quite high: more than a quarter of this sample $(27.5 \%)$ had diabetes. More than $61 \%$ of those with diabetes rated their oral health as fair/poor, a rate substantially higher than that of their non-diabetic counterparts $(49.2 \%)$ as well as that of the USA general population (30\%) [28]. However, at $61.1 \%$ and $62.5 \%$, the rate of dental service use was comparable in the groups with and without diabetes, respectively. It is noteworthy that nearly $40 \%$ of the group with diabetes had never visited a dental clinic in the past year and that their oral health needs were unattended. Given this group's known risks for oral complications and their negative evaluations of personal oral health condition, $[1-3,5]$ the underutilization of dental services among individuals with diabetes deserves further attention.

The findings from the multivariate models confirm the pattern observed in the descriptive and comparative analyses. The presence of diabetes increased the odds of reporting fair/poor oral health, and its impact remained significant after controlling for demographic, immigration-related, and health and access variables. On the other hand, the presence of diabetes had no significant impact on dental service use. Among the covariates considered, the variables representing lack of resources, such as unmarried status, low education, and no dental insurance coverage, were commonly associated with negative ratings of oral health and no use of dental care services. Female gender was associated with higher odds of having a positive oral health rating and of using dental care services. Both immigration-related 
Citation: Jang Y, Rhee MK, Jeong CH, Mulligan R, Kim (2019) Self-Rated Oral Health and Dental Service Use of Older Korean Americans with Diabetes. J Gerontol Geriatr Med 5: 034.

variables-length of stay in the USA and acculturation-were significant factors for self-rated oral health, promoting positive evaluations. For dental service use, significance was observed only in the length of stay: those who had stayed longer in the USA had higher odds of using dental care services. This finding is in line with previous studies suggesting that time in the USA allows immigrants to become familiarized with the USA healthcare system and to accommodate their healthcare needs $[29,30]$. Medical comorbidity significantly increased the odds of reporting negative oral health ratings but had no impact on dental service use.

The findings of the present study highlight the missing link between diabetes and dental service use. Those with diabetes demonstrated that their oral health needs were high but unattended. The fact that almost $40 \%$ of the individuals with diabetes had no dental visit in the past year invites further assessment of their knowledge and awareness of the importance of oral health and dental care in relation to diabetes. In our supplementary analysis, a majority $(84 \%)$ of the individuals with diabetes but without dental service use had no dental insurance. Beyond dental insurance, other variables that might facilitate or hinder access to dental care, such as transportation, medical communication skills, and oral health beliefs, also need to be considered. Given the medical-dental divide in the USA healthcare system,[13-15] our findings underscore the importance of involving both primary care physicians and dental care professionals in integrated care for oral health and diabetes.

Some limitations to the present study should be noted. Although we attempted to include diverse groups of older Korean Americans in terms of their socioeconomic status, cultural and linguistic levels, and geographic locations, generalizability of the findings is limited by our non-probability sampling method. Also, the study's cross-sectional design does not allow one to draw causal inferences among the variables. The use of a single item to assess self-rated oral health and dental service use is also a limitation. Future studies should include clinical oral examinations as well as detailed information on dental service use, such as the nature of visits (e.g., preventive care, acute care and limited prosthesis repairs), ethnic and linguistic concordance with care providers, and satisfaction with care received. Given that the presence of diabetes was not associated with dental service use despite its significant adverse effect on self-rated oral health, researchers should explore the intervening role of self-rated oral health and other factors (e.g., oral health literacy and social support) that may influence underlying mechanisms between diabetes and dental service use.

Despite these limitations, the present study has improved our understanding of oral health needs and dental care in older Korean Americans with diabetes, and it provides implications for health services and policies. Its findings highlight the importance of partnerships between dental and primary care providers, which can inform patients about the relationship between diabetes and oral health and lead to dental or medical treatment for prevention and better management. Overall, this study's findings underscore the importance of integrated healthcare systems to better manage oral health and diabetes care.

\section{Acknowledgement}

Data collection was supported by a grant from the National Institute on Aging (R01AG047106, PI: Yuri Jang, Ph.D.). The authors thank Drs. Kunsook Bernstein, SoonheeRoh, Soyeon Cho, Sanggon
Nam, and Seunghye Hong and community advisors for help with data collection. The authors report no conflicts of interest.

\section{References}

1. Huang DL, Chan KC, Young BA (2013) Poor oral health and quality of life in older U.S. adults with diabetes mellitus. J Am Geriatr Soc 61: 17821788 .

2. Lamster IB, Lalla E, Borgnakke WS, Taylor GW (2008) The relationship between oral health and diabetes mellitus. J Am Dent Assoc 139: 19-24.

3. Leite RS, Marlow NM, Fernandes JK, Hermayer K (2013) Oral health and type 2 diabetes. Am J Med Sci 345: 271-273.

4. López-Pintor RM, Casañas E, González-Serrano J, Serrano J, Ramírez L, et al (2016) Xerostomia, Hyposalivation, and Salivary Flow in Diabetes Patients. J Diabetes Res 4372852: 1-15.

5. Sandberg GE, Sundberg HE, Fjellstrom CA, Wikblad KF (2000) Type 2 diabetes and oral health: A comparison between diabetic and non-diabetic subjects. Diabetes Res ClinPract 50: 27-34.

6. Taylor GW, Borgnakke WS (2008) Periodontal disease: Associations with diabetes, glycemic control and complications. Oral Dis 14: 191-203.

7. Teshome A, Yitayeh A (2016) The effect of periodontal therapy on glycemic control and fasting plasma glucose level in type 2 diabetic patients: Systematic review and meta-analysis. BMC Oral Health 17: 31.

8. Teeuw WJ, Gerdes VE, Loos BG (2010) Effect of periodontal treatment on glycemic control of diabetic patients: A systematic review and meta-analysis. Diabetes Care 33: 421-427.

9. Darling-Fisher CS, Kanjirath PP, Peters MC, Borgnakke WS (2015) Oral Health: An Untapped Resource in Managing Glycemic Control in Diabetes and Promoting Overall Health. J Nurse Pract 11: 889-896.

10. Gillis MR (2010) A place for oral health in diabetes management. J Can Dent Assoc 76: 1-3.

11. Bowyer V, Sutcliffe P, Ireland R, Lindenmeyer A, Gadsby R, et al. (2011) Oral health awareness in adult patients with diabetes: a questionnaire study. Br Dent J 211: 12.

12. Yuen HK, Wolf BJ, Bandyopadhyay D, Magruder KM, Salinas CF, et al. (2009) Oral health knowledge and behavior among adults with diabetes. Diabetes Res ClinPract 86: 239-246.

13. Atchison KA, Rozier RG, Weintraub JA (2018) Integration of Oral Health and Primary Care: Communication, Coordination, and Referral. NAM Perspectives: 1-12.

14. Lee JS, Somerman MJ (2018) The Importance of Oral Health in Comprehensive Health Care. JAMA 320: 339-340.

15. Mertz EA (2016) The Dental-Medical Divide. Health Aff (Millwood) 35: 2168-2175.

16. Pew Research Center (2017) Koreans in the U.S. Fact Sheet. Pew Research Center, Washington, D.C., USA.

17. U.S. Census Bureau (2018) Selected Characteristics of the Native and Foreign-Born Populations: 2012-2016 American Community Survey 5-Year Estimates. U.S. Census Bureau, Maryland, USA.

18. Kwok D (2013) President's Advisory Commission on Asian Americans and Pacific Islanders Meet in Washington, D.C., USA.

19. Jang Y, Yoon H, Park NS, Chiriboga DA, Kim MT (2014) Dental care utilization and unmet dental needs in older Korean Americans. J Aging Health 26: 1047-1059. 
Citation: Jang Y, Rhee MK, Jeong CH, Mulligan R, Kim (2019) Self-Rated Oral Health and Dental Service Use of Older Korean Americans with Diabetes. J Gerontol Geriatr Med 5: 034.

20. Kim MT, Kim KB, Huh B, Nguyen T, Han HR, et al. (2015) The Effect of a Community-Based Self-Help Intervention: Korean Americans With Type 2 Diabetes. Am J Prev Med 49: 726-737.

21. Kim MT, Kim KB, Ko J, Jang Y, Levine D, et al. (2016) Role of depression in diabetes management in an ethnic minority population: a case of Korean Americans with type 2 diabetes. BMJ Open Diabetes Res Care 5: 1-9.

22. Yoon KH, Lee JH, Kim JW, Cho JH, Choi YH, et al. (2006) Epidemic obesity and type 2 diabetes in Asia. Lancet 368: 1681-1688.

23. Wu B, Plassman BL, Liang J, Remle RC, Bai L, et al. (2011) Differences in self-reported oral health among community-dwelling black, Hispanic, and white elders. J Aging Health 23: 267-288.

24. Institute of Medicine (2018) Advancing Oral Health in America. The National Academies Press, Washington, D.C., USA.

25. American Diabetes Association (2018) Economic costs of diabetes in the U.S. in 2017. Diabetes Care 42: 917-928.
26. Jang Y, Kim G, Chiriboga D, Kallimanis B (2007) A Bidimensional Model of Acculturation for Korean American Older Adults. J Aging Stud 21: 267-275.

27. IBM Corp (2017) IBM SPSS Statistics for Windows. Version 25.0. 25 ed. IBM Corp, New York, USA.

28. American Dental Association (2015) Oral Health and Well-Being in the United States. Health Policy Institute, American Dental Association, New York, USA.

29. Jang Y, Yoon H, Rhee MK, Park NS, Chiriboga DA, et al. (2019) Factors associated with dental service use of older Korean Americans. Community Dent Oral Epidemiol 47: 340-345.

30. Luo H, Wu B (2016) Acculturation and Dental Service Use Among Asian Immigrants in the U.S. Am J Prev Med 51: 939-946. 


\section{II \\ HERALD}

Journal of Anesthesia \& Clinical Care

Journal of Addiction \& Addictive Disorders

Advances in Microbiology Research

Advances in Industrial Biotechnology

Journal of Agronomy \& Agricultural Science

Journal of AIDS Clinical Research \& STDs

Journal of Alcoholism, Drug Abuse \& Substance Dependence

Journal of Allergy Disorders \& Therapy

Journal of Alternative, Complementary \& Integrative Medicine

Journal of Alzheimer's \& Neurodegenerative Diseases

Journal of Angiology \& Vascular Surgery

Journal of Animal Research \& Veterinary Science

Archives of Zoological Studies

Archives of Urology

Journal of Atmospheric \& Earth-Sciences

Journal of Aquaculture \& Fisheries

Journal of Biotech Research \& Biochemistry

Journal of Brain \& Neuroscience Research

Journal of Cancer Biology \& Treatment

Journal of Cardiology: Study \& Research

Journal of Cell Biology \& Cell Metabolism

Journal of Clinical Dermatology \& Therapy

Journal of Clinical Immunology \& Immunotherapy

Journal of Clinical Studies \& Medical Case Reports

Journal of Community Medicine \& Public Health Care

Current Trends: Medical \& Biological Engineering

Journal of Cytology \& Tissue Biology

Journal of Dentistry: Oral Health \& Cosmesis

Journal of Diabetes \& Metabolic Disorders

Journal of Dairy Research \& Technology

Journal of Emergency Medicine Trauma \& Surgical Care

Journal of Environmental Science: Current Research

Journal of Food Science \& Nutrition

Journal of Forensic, Legal \& Investigative Sciences

Journal of Gastroenterology \& Hepatology Research

Journal of Gerontology \& Geriatric Medicine

Journal of Genetics \& Genomic Sciences

Journal of Hematology, Blood Transfusion \& Disorders

Journal of Human Endocrinology

Journal of Hospice \& Palliative Medical Care

Journal of Internal Medicine \& Primary Healthcare

Journal of Infectious \& Non Infectious Diseases

Journal of Light \& Laser: Current Trends

Journal of Modern Chemical Sciences

Journal of Medicine: Study \& Research

Journal of Nanotechnology: Nanomedicine \& Nanobiotechnology

Journal of Neonatology \& Clinical Pediatrics

Journal of Nephrology \& Renal Therapy

Journal of Non Invasive Vascular Investigation

Journal of Nuclear Medicine, Radiology \& Radiation Therapy

Journal of Obesity \& Weight Loss

Journal of Orthopedic Research \& Physiotherapy

Journal of Otolaryngology, Head \& Neck Surgery

Journal of Protein Research \& Bioinformatics

Journal of Pathology Clinical \& Medical Research

Journal of Pharmacology, Pharmaceutics \& Pharmacovigilance

Journal of Physical Medicine, Rehabilitation \& Disabilities

Journal of Plant Science: Current Research

Journal of Psychiatry, Depression \& Anxiety

Journal of Pulmonary Medicine \& Respiratory Research

Journal of Practical \& Professional Nursing

Journal of Reproductive Medicine, Gynaecology \& Obstetrics

Journal of Stem Cells Research, Development \& Therapy

Journal of Surgery: Current Trends \& Innovations

Journal of Toxicology: Current Research

Journal of Translational Science and Research

Trends in Anatomy \& Physiology

Journal of Vaccines Research \& Vaccination

Journal of Virology \& Antivirals

Archives of Surgery and Surgical Education

Sports Medicine and Injury Care Journal

International Journal of Case Reports and Therapeutic Studies

Submit Your Manuscript: http://www.heraldopenaccess.us/Online-Submission.php 